Traditional Medicine and Modern Medicine

Vol. 1, No. 1 (2018) 1-10

(C) Institutes of Integrative Medicine, Fudan University and

World Century Publishing Corporation

DOI: $10.1142 /$ S2575900018100018

\title{
Philosophical thinking of Chinese Traditional Medicine
}

\author{
Jingcheng Dong*, Linwei Lu, Jingjing Le, Chen Yan, Hongying Zhang and Lulu Li \\ Department of Integrative Medicine, Huashan Hospital, Fudan University \\ 12 Middle Urumqi Road, Shanghai 200040, P. R. China \\ Institute of Theories and Application, The Academy of Integrative Medicine \\ Fudan University, Shanghai 200032, P. R. China \\ jcdong2004@hotmail.com
}

Received 23 August 2017; Revised 15 December 2017; Published 8 March 2018

\begin{abstract}
Traditional medicine is often an integration of ancient philosophy, clinical experiences, primitive knowledge of medicine, regional cultures and religious beliefs. Chinese Traditional Medicine (CTM) is the general appellation of all the traditional medicines of different ethnicities in China, which share great similarities of basic concept and philosophical basis, and conform to the development of empirical medicine, among which the medicine of Han ethnicity (Han medicine) is the most mature. The development of CTM is totally different from that of modern medicine, always revolving around the center of disease diagnosis and treatment, establishing the core theoretical system of Yin and Yang, Five Elements, Zang and Fu and Humoralism with the theoretical foundation of ancient Chinese philosophy, which represents the highest achievement of worldwide empirical medicine and philosophy form at that time. In general, the basic structure of CTM mainly consists of three parts as follows: the part that has already reached consensus with modern medicine, the part that is unconsciously ahead of modern medicine, and the part that needs to be reconsidered or abandoned.
\end{abstract}

Keywords: CTM; modern medicine; classical philosophy; trichotomy; five key elements; inflammatory/antiinflammatory imbalance (IAII); inflammatory/anti-inflammatory balance adjustment mechanism (IAIBAM).

\section{Introduction}

There exist many inaccurate concepts and understandings that have influenced the development of Chinese Traditional Medicine (CTM). For example, people have always faced confusions such as what Han medicine is, what TCM is, what CTM is, what traditional medicine is, what national medicine is, what modern medicine is, and how to correctly understand traditional medicine. These confusions have caused obvious problems like the sense of identity of Chinese nation from the macro points of view, and the level of diagnosis and treatment of specific patients from the micro points of view. Therefore, people have the responsibility to realize and solve them gradually. As we all know, traditional medicine is often a mixture of Five key elements, including ancient philosophy, clinical experiences, primitive knowledge of medicine, regional cultures, and religious beliefs, while classical philosophy of different nationalities is the basis to framework traditional medical theories. Thus, it is of great significance to further think about the philosophical foundation and basic framework of CTM. Hu Shi once said in The Outline of the Chinese Philosophy History that whenever researching on the critical problems of lifetime, think fundamentally, find a fundamental solution, and that's called philosophy. Meanwhile, the thinking on philosophical basis and basic framework of CTM will be helpful to fundamentally solve all the confusions we are facing in the field of CTM.

\section{The Basic Situation of CTM}

CTM is a medical system of unique theoretical style, gradually formed in long-term medical and life practice with constant accumulation and repeated summarization. CTM is

\footnotetext{
*Corresponding author.
} 
the general appellation of all the nationalities/ethnicities in China, including Han medicine, Uyghur medicine, Tibetan medicine, Mongolian medicine, Dai medicine, Hui medicine, Miao medicine and Zhuang medicine. In the field of CTM, Han medicine has the most influence in China and worldwide due to its large population, earliest text records, long history and splendid culture, relatively more perfect theoretical system of traditional medicine, and richer clinical practice. During the Opium War in 1840s, the so-called Western medicine (modern medicine) at the time was introduced into China, while Han medicine was given the alias "Chinese medicine" so as to differentiate it from the Western medicine that is called modern medicine today. Each traditional medicine of different ethnicities usually has its own extensive and profound basis.

\section{Han medicine}

Han medicine has basically established its own unique theoretical system in the Spring-Autumn and Warring States period, with theoretical basis on classical Chinese philosophical concepts like Qi (Vitality), Spirit (Shen), Deficiency, Yin and Yang and Five Elements. Its way of thinking reflects the holistic, organic and dynamic thinking of classical Chinese philosophy, while its physiological and pathological basis is based on Zang and $F u$ as well as Meridians and Collaterals. Its pioneers are Hua Tuo, Bian Que and Zhang Zhongjing, and representative books are Shennong's Classic of Materia Medica, Classic of Difficulties, Shennong's Herbal Classic, Compendium of Materia Medica, and Treatise on Febrile Diseases. In the theoretical system of CTM, it lacks the insights into anatomy, but emphasizes on the balance of Yin and Yang and etiologically on Six Pathogenic Factors and Seven Emotions, pays attention to the overall concepts, Syndrome differentiation and treatment, and treats different diseases with the same method as well as the same disease with different methods, etc. Together with Four Diagnostic Methods of inspection (wang), auscultation and olfaction (wen), inquiry (wen), pulse-taking and palpation (qie), Eight Therapeutic Methods are well established including sweating (han), vomiting (tu), purging (xia), harmonizing (he), warming (wen), clearing (qing), reducing (xiao) and tonifying $(b u)$. Furthermore, the prescription of herbal medicines is based on the principle of Monarch (Jun), Minister (Chen), Adjuvant (Zuo) and Assistant (Shi) Drugs.

\section{Uyghur medicine}

Uyghur medicine has a history of hundreds of years. It is rooted in the theory of Four Temperaments and Humoralism of Greek philosophy, while absorbing the essence of Arabian and Han medicine. The pioneers are Kumarajiva, al-Fārāb̄̄ and ibn-Sinna. The representative books are Empirical Prescriptions for Diseases and The Canon of Medicine, which have deep understandings on anatomy. Besides the emphasis on four diagnostic methods, it has even adopted new diagnostic methods on urine, stool and sputum in terms of inspection.

\section{Tibetan medicine}

Tibetan medicine has a history of hundreds of years that is also theoretically based on Yin and Yang and Four Principles, whose pioneers are Yu tuo·Yun deng gong bu, Bi qi lie gong, $\mathrm{Wu}$ ba qu sang, and the representative book is The Four Medical Tantras (i.e. rGyud bzhi). It emphasizes Zang and $\mathrm{Fu}$, which is the center of human body. Specifically, a human body is made up of Three Factors including Long (Qi), Chiba (Fire) and Peigen (Water and Earth), which dominate and regulate Seven Materials like essence of food or chyle, blood, muscle, fat, bone, marrow and vital essence, three excretions like sweat, stool and sputum, 360 bones, nine holes in the body and black and white pulses. Like Uyghur medicine, it puts special emphasis on urinalysis in addition to four diagnostic methods, and has the characteristics of Syndrome differentiation from the aspect of diagnosis and treatment. Also, it prescribes according to the taste before and after digestion and function of medicine, and pays attention to adjusting doses for better efficacy and prescribing appropriately.

\section{Mongolian medicine}

Mongolian medicine, with a history of hundreds of years, experienced rapid development from the $13^{\text {th }}$ century and established its unique system around the $18^{\text {th }}$ century. It is based on the theories of Yin and Yang, Five Elements and Five Principles. Its pioneers are Luo bu sang dan jin zha la sang, Yi xi ba la zhu er and Zhan bu la dao er ji, and its representative books are Four Dews, Canon of Mongolian Medicine, and Sea of Prescriptions, which have a rather profound understanding of anatomy. Apart from the diagnosis of inspection, inquiry and pulse-taking, it also puts emphasis on palpation, auscultation and olfaction as well as on the urine examination and the differentiation of Cold and Heat Syndromes.

\section{Dai medicine}

Dai medicine has a history of hundreds of years. After the Middle Ages, it was centered on ancient materialism and Theravada Buddhist philosophy of naive dialectics. Its pioneers are Wa ji la bie, Gu ma la bie, Ya dang na mo ya ba dun, and its representative books are Luo ge ya tan, Ba la ma ta tan, Ga ya shan ha ya, Dang ha ya long, which have a rather thorough understanding of anatomy, putting emphasis on the differentiation of Four Elements like Wind (Feng), Fire 
(Huo), Water (Shui) and Earth (Tu), Five Skandhas like Material (Se), Feeling (Shou), Association (Xiang), Mentality (Xing) and Perception (Shi). Rich treatments lead to different prescriptions including simple and complex prescriptions.

\section{Hui medicine}

Hui medicine originated from the $13^{\text {th }}$ century, focusing on the holistic thinking and the theory that human is an integral part of nature, as well as on the Theories of Vitality, Yin and Yang and Seven Elements. Its pioneers are Ma Shikui and Ma Jinliang, and its representative books are Hui Prescriptions and Principles of Healthy Diets. Although Hui medicine lacks the knowledge of anatomy, it puts emphasis on quality differentiation, and subsequently flexible and rich treatment based on Syndrome differentiation.

\section{Miao medicine}

Miao medicine has a history of hundreds of years, which is the combination of theology and witchcraft without sufficient insights into anatomy, and also has been influenced by Han medicine to a large extent. It divides all the diseases into two categories as Cold and Heat Diseases, which has quite rich treatments like treating Heat Diseases via Cold methods and treating Cold Diseases via Heat methods. In terms of the number of drugs in one prescription, it has the features of prescribing in an odd number or by means of three-in-one.

\section{Zhuang medicine}

Zhuang medicine originated from primitive society and developed rapidly after Tang and Song Dynasty, which is theoretically based on Yin and Yang, heaven-and-human nature view of Three-Qi Synchronization. Its pioneer is Luo Jia' an, and its representative books are Acupuncture Diagram of Measles Syndromes and The Medical History of Zhuang Nationality, which regards Viscera, Qi (Vitality), blood, bone and muscle as the main material basis of human body. Those in the intracranial, pleural and abdominal cavities or rather independent entities can be called Viscera, but Zhuang medicine does not make distinctions between Zang and Fu. It puts emphasis on eye diagnosis, combines disease and Syndrome differentiation, and has the regular usage of animal medicine.

By briefly comparing the basic concepts, history, philosophical basis, pioneers and representative books, anatomy, physiology, etiology, pathogenesis, diagnosis, therapeutic principles and prescription characteristics of traditional medicines of different ethnicities in China, it is easy to find great similarities in considerable aspects. Most have the characteristic of general/holistic regulation in accordance with experienced medicine, among which Han medicine is the most mature.

\section{The Philosophical Basis of CTM}

In summary, it is obvious that all traditional medicines of different ethnicities have long histories, profound theories, flexible techniques and diagnosis, exquisite prescriptions, distinctive and unique systems, which contribute greatly to the prosperity of all ethnicities in China. But we should also admit that the development of CTM is totally different from that of modern medicine. CTM always focuses on Syndrome differentiation and solves practical problems, follows the

Table 1. The basic situation of various Chinese Traditional Medicines.

\begin{tabular}{|c|c|c|c|}
\hline Categories & Theoretical Basis & Pioneers & Representative Books \\
\hline Han Medicine & $\begin{array}{l}\text { Yin and Yang, Five Elements, } \\
\text { Zang and } F u, \text { Humoralism }\end{array}$ & $\begin{array}{l}\text { Hua Tuo, Bian Que, Zhang } \\
\text { Zhongjing }\end{array}$ & $\begin{array}{l}\text { Inner Canon of Yellow Emperor, Classic of } \\
\text { Difficulties, Shennong's Classic of Materia } \\
\text { Medica, Compendium of Materia Medica, } \\
\text { Treatise on Febrile Diseases }\end{array}$ \\
\hline Uyghur Medicine & $\begin{array}{l}\text { Four Temperaments and } \\
\text { Humoralism }\end{array}$ & Kumarajiva, al-Fārābī, ibn-Sīna & $\begin{array}{l}\text { Empirical Prescriptions for Diseases, The Canon } \\
\text { of Medicine }\end{array}$ \\
\hline Tibetan Medicine & Yin and Yang, Four Principles & $\begin{array}{l}\text { Yu tuo· Yun deng gong bu, Bi qi lie } \\
\text { gong, Wu ba qu sang }\end{array}$ & The Four Medical Tantras (rGyud bzhi) \\
\hline Mongolian Medicine & $\begin{array}{l}\text { Yin and Yang, Five Elements, Five } \\
\text { Principles }\end{array}$ & $\begin{array}{l}\text { Luo bu sang dan jin zha la sang, Yi xi } \\
\text { ba la zhu er, Zhan bu la dao er ji }\end{array}$ & $\begin{array}{l}\text { Four Dews, Canon of Mongolian Medicine, Sea } \\
\text { of Prescriptions }\end{array}$ \\
\hline Dai Medicine & $\begin{array}{l}\text { Ancient materialism, Theravada } \\
\text { Buddhist philosophy of naive } \\
\text { dialectics }\end{array}$ & $\begin{array}{l}\text { Wa ji la bie, Gu ma la bie, Ya dang na } \\
\text { mo ya ba dun }\end{array}$ & $\begin{array}{l}\text { Luo ge ya tan, Ba la ma ta tan, Ga ya shan ha ya, } \\
\text { Dang ha ya long }\end{array}$ \\
\hline Hui Medicine & $\begin{array}{l}\text { The holistic thinking, the } \\
\text { Theories of Vitality, Yin and } \\
\text { Yang, Seven Elements }\end{array}$ & Ma Shikui, Ma Jinliang & Hui Prescriptions, Principles of Healthy Diets \\
\hline Miao Medicine & $\begin{array}{l}\text { Theology, witchcraft, Han } \\
\text { medicine }\end{array}$ & l & / \\
\hline Zhuang Medicine & $\begin{array}{l}\text { Yin and Yang, Three-Qi } \\
\text { Synchronization }\end{array}$ & Luo Jia'an & $\begin{array}{l}\text { Acupuncture Diagram of Measles Syndromes, } \\
\text { The Medical History of Zhuang Nationality }\end{array}$ \\
\hline
\end{tabular}


wisdom of ancient times, and establishes central medical theories of Yin and Yang, Five Elements and Humoralism with the theoretical basis of ancient simple materialism philosophy. CTM system, or Chinese philosophy, represents the highest achievements of the world's empirical medicine and philosophy at that time. Although experience and techniques prevail in this system, it is hard to find its contribution to basic science based on reductionism. A common characteristic of ancient simple materialism philosophy is that the diversity of nature cannot be generally abstracted as objective material, and vivid and specific objects along with their forms of movement are needed to describe and explain the real world. The so-called monism, dualism and pluralism then come into being because the universe can be explained by one objective and its movement, and others, however, might be explained more specifically and sophisticatedly. These philosophical theories exist not only in ancient Greece, but also in CTM of ancient China at the same time. Notably, be it Han medicine or other traditional medicines mentioned above, they almost invariably choose pluralism philosophy when constructing their theoretical systems. Han and Mongolian medicine have adopted Five Elements while other traditional medicines, such as Uyghur and Tibetan medicine, prefer Four Elements. Comparing the essence of Five Elements with that of Four Elements, it is not difficult to find that their similarities outweigh their differences. Firstly, both theories were generated at the same stage of human developmental history, also on the same level of civilization development. Secondly, they have shared lots of important similarities in the views and forms, while the differences are that the contents of Five Elements are more abundant than those of Four Elements. The reason why various ancient traditional medicines chose multiple philosophies is that it can provide a kind of dynamic model of formalization, while monism and dualism are too abstract to be particularly applied in the field of natural science. That is to say, pluralism can provide not only philosophical thinkings, but also basic framework of constructing the theoretical systems of various traditional medicines.

The Viscera Theory of Han medicine was once called Visceral Manifestation in ancient times, meaning viscera are stored inside but can be observed from outside through physiological and pathological changes. The Viscera Theory is the phenomenological theory constructed from external phenomenon of the body, but neither from the anatomy of internal organs nor the form of physiological structureactivity relationship. Each national traditional medicine has established its own theory on the basis of phenomenology, which has been improved feasibly to a certain extent. Influenced by ancient simple materialism, traditional medicines including Han medicine have established their own phenomenology based on experiences. On the other hand, fragmented experiences are abstracted, formalized and standardized, evolving into various theories so as to preserve, develop, communicate and teach experience medicine. Admittedly, there are fallacies among many of such experiences and theories, while some of them might contain the laws of science. The Viscera Theory originated during the collapse of the slavery system and start of the feudal period of China, while relatively complete Humoralism originated at the heyday of ancient Greek slavery. If we view from the specific time point, the latter was a little earlier than the former. The backgrounds of these two, however, had several important similarities, first of which was that both of them were formed after the shake of ghosts pathogenicity (reconstruction of medical theories after religious or witchcraft medicine), second of which was that both of them generated at the most developed countries of ancient civilization at that time, third of which was that both of them were the combination of ancient simple materialism and limited clinical experience, belonging to experience medical theories based on ancient philosophical concept speculation. Therefore, the formation of Humoralism and the Viscera Theory in CTM has its historical inevitability.

Ancient knowledge of anatomy, physiology, pathology and pharmacology was extremely poor and simple, but in order to explain various pathological and physiological phenomena of human body and teach limited clinical experience, they had to establish some kind of theories and systems of medical knowledge with the help of philosophical speculation. In fact, there was a huge span from philosophy to specific categories of natural science, among which lots of knowledge is needed to fulfill it. So, in essence, ancient theoretical systems of experience medicine were the replica of pluralism philosophy. However, this kind of replica might contain high rationality. The Viscera Theory and Humoralism were just established in this way, such as Han medicine replacing Wood (Mu), Fire (Huo), Earth (Tu), Metal (Jin) and Water (Shui) with Liver (Gan), Heart (Xin), Spleen (Pi), Lung (Fei) and Kidney (Shen) respectively. Viscera were given the attributes and functions of Five Elements, and furthermore, the dynamic relationship like that among Five Elements. This kind of visceral structure, function and relationship then became the basic model of body constitution and function. All the physiological or pathological processes could be described and explained in this simple functional model. Humoralism in Uyghur medicine replaced Fire (Huo), Qi (Vitality), Water (Shui), and Earth (Tu) in Tibetan medicine with Choleric, Melancholic, Sanguine, and Phlegmatic Temperaments. Meanwhile, it also introduced the characteristics and relationships between various bodily fluids, and subsequently interpreted various pathological and physiological processes of human body by fluid ratio, distribution and quality. Doubtlessly, the Viscera Theory also puts emphasis on the importance of blood and fluid, making tight 
combination of visceral and humoral metabolism and function. Humoralism was no exception, which regarded that fluid was generated from viscera, put emphasis on viscera, and made combination of these two to recognize and explain complicated pathological and physiological phenomena. All in all, these recognitions and explanations from our ancestors focused on the guiding ideology of dialectical materialism philosophy that the universe was developing, changing and unifying of opposites. All the traditional medicines centered on the Viscera Theory and Humoralism to establish their own theories, reflecting that scientific and cultural level, subjected to the cognition of ancient human lives at the time, still remained in the primitive stage. Theoretically speaking, there was no significant difference between the Viscera Theory and Humoralism, as both belonged to the simple phenomenological theory, having a simple holistic view and balanced concept of etiology, pathology and prevention. The content of the Viscera Theory was more plentiful and specific than that of Humoralism so that the practical value of the Viscera Theory surpassed that of Humoralism. It made combination of visceral function with external variables such as symptoms and signs of human bodies or with intermediate variables such as Yin, Yang, Qi (Vitality), Blood, Essence and Fluid respectively, so as to detect various external manifestations of human body (including symptoms and signs) through state changes of visceral function or vice versa. Subsequently, the treatment, therapy, traditional drug efficacy and efficacy determination were brought into a unified theoretical system centralized by the Viscera Theory and Humoralism, so as to realize the integration of diseases, Syndromes, theories, diagnostics, prescriptions and drugs.

CTM represented by Han medicine had mainly developed into clinical empirical medicine, not experimental or basic medicine, but it did reach the highest realm of human empirical medicine. Some of the precious experience has been proved to be unanimous to the recognition of modern medicine and explained by modern medicine. Some might, to some extent, guide the direction of the advancement of modern medicine. However, these empirical medicines generated thousands of years ago inevitably have their limitations due to the era and culture, among which abundant understandings of the era and culture also existed in addition to the precious experience.

\section{The Basic Structure of CTM}

As mentioned before, CTM constructed with the help of clinical practice and classical philosophy is substantially the most advanced, complete and experienced medicine in the world, whose vast system fills with practical medical technology and experience gradually identified by modern medicine. It contains some foreseeing outlook and germs for future development and direction, and possesses simple understanding or even mistakes associated with the specific era and culture. Therefore, as a whole, the basic structure of CTM could be probably constituted of the following three parts: the part that has already reached consensus with modern medicine, the part that is unconsciously ahead of modern medicine, and the part that needs to be reconsidered or abandoned.

\section{The part that has already reached consensus with modern medicine}

The so-called Western medicine (modern medicine) was introduced into China in about the $16^{\text {th }}$ century, but had little influence then. After the Opium War (in 1840s), it gradually developed in China. Therefore, during the Chinese cultural history before the Opium War, CTM was the main strength of Chinese health care all the time, the key of which was its efficacy. Genuine knowledge comes from practice, which means a large number of practical cases decorated simple theories with a strong foundation. After the establishment of People's Republic of China (new China), the development of CTM and modern medicine entered a new stage. With the further influence of modern medicine, especially the development of the so-called integrative medicine in the late 1950s, CTM, especially Han medicine, demonstrated some connotations of modern life science in theories, treatments, diagnosis, technologies and prescriptions so that the basic structure of CTM turned clear. One of these important constitutions is the part having already formed consensus with modern medicine, involving many basic and clinical links of CTM. Take several theories of Han medicine and significant concepts for example, research on Kidney (Shen) Essence by Academician Shen Ziyin indicated that Kidney (Shen) Deficiency Syndrome is equivalent to premature aging or aging changes of modern medicine to a large extent. ${ }^{1}$ Systematic research on treating coronary heart disease with the method of promoting blood circulation and removing blood stasis by Academician Chen Keji scientifically demonstrated the mechanism and the essence of Blood Stasis, which had won the consensus of CTM and modern medicine experts.

Inspection (wang) is a kind of method to determine illness condition by observing the changes of systemic or local expression, color, shape and state. Usually, inspection (wang) contains not only observing coated tongue, but also observing external features of the patients from where internal illness conditions can be detected. The observations on patients' expression, color, shape and state are particularly important, which have reached consensus with the diagnostics of modern medicine in many aspects. For example, CTM regards pale lips as important performance of $Q i$ and Blood Deficiency, while modern medicine similarly regards 
that pale lips may be one of the important clues of diagnosing anemia. CTM regards cyanosis as the manifestation of Blood Stasis, while modern medicine regards it as an important basis of diagnosing hypoxemia and hypercapnia. Auscultation and olfaction (wen) include listening and smelling, which have also reached consensus with the clinical diagnostics of modern medicine. For example, loud, sonorous and forceful talks of patients can be judged as full of essence and energy or not-so-severe conditions by both CTM and modern medicine. On the contrary, if patients speak feebly and weakly or even fail to talk, CTM regards it as Qi Deficiency, while modern medicine regards it the result of organ function failure or severe body consumption caused by many serious diseases. Smelling includes the odor of secretion in the mouth and body. CTM regards that it could be used in Syndrome differentiation, while modern medicine also regards it highly related to diseases. For example, the rotten apple smell in a patient' body indicates the possibility of diabetic ketoacidosis. Inquiry (wen) mainly refers to asking patients' physical and mental feelings, discomfort and the incidence and severity of diseases by asking life history, family history, past medical history, onset time and symptoms at present, which has already been widely applied in CTM and modern medicine. Pulse-taking and palpation (qie) mean that doctors touch or press some parts of a patient's body through hands or fingertips, getting the information about the changes of pulse, the characteristics of lumps in the chest or abdomen, the categories of swelling in the skin, the temperatures of limbs or the pain area, etc. The comprehensive application of four diagnostic methods makes the diagnosis of diseases. Pulse-taking is particularly an indispensable basic method in CTM clinical diagnosis, which could reveal a great deal of information of diseases and Syndromes, and is agreed by modern medicine in the value of knowing heart rate, cardiac rhythm, vascular filling and hardening degree.

Take several classical prescriptions of CTM for example, many diseases that can be treated by classical prescriptions can be basically equal to some specific diseases and conditions recognized by modern medicine at present: (1) Pure Ginseng Decoction (Du shen tang) could be used in hemorrhagic or cardiogenic shock, as ginseng helps to nourish $Q i$ (Vitality) and enrich Blood..$^{2-4}$ (2) Decoction for Treating Yang Exhaustion (Si ni tang) could be used in a variety of shock cases with the help of dispelling Cold and reviving Yang by Radix Aconiti Carmichaeli (Fu zi), dried Rhizoma Zingiberis (Gan jiang) and prepared Radix Glycyrrhizae (Gan cao). (3) Decoction for Strengthening the Spleen-Yang (Zhen wu tang) could be seen in cardiac and renal edema of modern medicine with the help of warming Yang and inducing diuresis by Poria Cocos (Fu ling), Herbaceous Peony (Shao yao), Rhizoma Atractylodis Macrocephalae (Bai zhu), Zingiber Officinale Roscoe (Sheng jiang) and Radix Aconiti
Carmichaeli (Fu zi). (4) Jade Screen Powder (Yu ping feng san) could invigorate Spleen (Pi), strengthen Defensive Exterior, and act as an antiperspirant. It is called the Chinese immunomodulator and is also applied in enhancing body immunity currently. ${ }^{5,6}$ Meanwhile, lots of Chinese medicines, no matter a single drug or prescription, show quite good and stable efficacy from thousands years of repeated use. With the development of modern medicine, the possible functional links and Syndromes intervened by these Chinese medicines become increasingly clear, and gradually consensus is reached with modern medicine. For example, Ephedra Vulgaris (Ma huang) treating asthma could be used in inducing perspiration and dispelling cold, dispersing Lung-Qi and relieving asthma as well as inducing diuresis and alleviating edema. Many CTM classical prescriptions treating asthma usually use Ephedra Vulgaris (Ma huang) as the Monarch Drug (Jun yao), whose modern pharmacological research has demonstrated that ephedrine, the main bioactive ingredient of Ephedra Vulgaris (Ma huang) belonging to alkaloids, is the sympathomimetic drug that acts on $\beta$-energic receptor, having an obvious relaxation effect on airway smooth muscle, especially when there is bronchial spasm. ${ }^{7,8}$ In CTM, Flos Daturae (Yang jin hua) has the function of relieving a range of symptoms including cough, asthma, convulsion, also having anesthesia, analgesia and spasmolysis effects. Modern research has shown that the main bioactive ingredient of Flos Daturae (Yang jin hua) is scopolamine that has the function of sedation, which can block the muscarinic acetylcholine receptor, generating atropine-like effects. Furthermore, this so-called part having already reached consensus with modern medicine can also be found in CTM surgery, such as in the orthopedics field represented by Han medicine.

\section{The part that is unconsciously ahead of modern medicine}

CTM is a practical medicine based on experience, the theoretical system of which was established on ancient simple materialism philosophy. It mostly adopted diagnosis and treatment methods based on Syndrome differentiation and applied natural means to treat diseases, but did not emphasize the accordance with science and technology at the same time. Different from the concept that nature that cannot be studied will not be conquered by modern medicine, CTM emphasized on holism, without requiring synchronization with the development of science at the same time, which has the understandings unconsciously beyond the era and culture. CTM has always guided modern medicine in the aspect of medical theory thinking. For example, CTM has the saying that man corresponds with nature, which is a unified view of organisms and the environment they live in. The so-called theories of chronobiology and biological clock (the discoveries 
were awarded the 2017 Nobel Prize), various rhythms led by hormone secretion, and the application of different therapies in different regions proposed by modern medicine reflect the theories of correspondence between man and nature. CTM has the viewpoints of the unity of opposites and dynamic balance, which increasingly shows that universal laws exist in biological phenomena. Although modern science also focuses on those points of view mentioned above, it is different from CTM in the way that CTM always takes this as its basic thought and theory. So CTM and modern medicine vary in the viewpoints of consciousness.

Our research ${ }^{9-11}$ found that chronic diseases involving Kidney (Shen) and the theory that fear would impair Kidney (Shen) had a profound modern scientific connotation. Lots of diseases, such as chronic airway inflammatory diseases that repeatedly recur, usually exacerbate the pathological changes of stress and anti-inflammatory system of HypothalamusPituitary-Adrenal (HPA) axis. Research has shown that various cytokines like IL- 6 and TNF- $\alpha$ could stimulate the synthesis of cortical hormone by up-regulating HPA axis when there is chronic inflammation, while cortical hormone could reduce the secretion of inflammatory cytokines, which is a negative feedback resulting in decreased secretion of cortical hormone to remove the inhibition to immune cells, increasing the secretion of inflammatory cytokines, then promoting the synthesis of cortical hormone. But in the course of time, there will be disorders that induce a vicious cycle, resulting in a serious inflammatory/anti-inflammatory imbalance (IAII), which causes disease protraction and finally the manifestation of Lung (Fei) - Kidney (Shen) Deficiency, or the so-called disease occurrence from Lung (Fei) to Kidney (Shen) (Fig. 1). Meanwhile, researchers have also shown that some negative emotions like fear and depression will cause repeated stress, and then dysfunction of HPA axis for a prolonged period, which leads to the IAII so that the so-called Kidney (Shen) Deficiency Syndrome would emerge. Take diagnosis and treatment for another example, the condition and

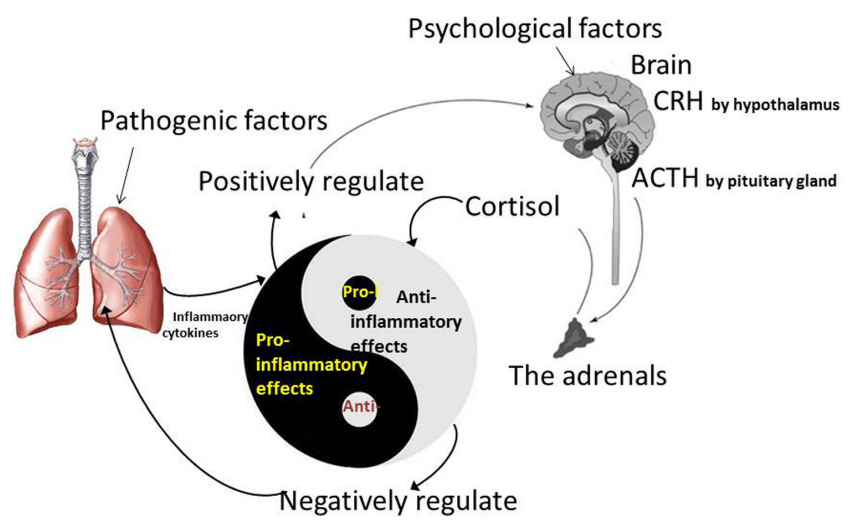

Figure 1. Cross talk between inflammatory and anti-inflammatory mechanism. disease of chronic inflammation have the CTM characteristics of homotherapy for heteropathy, representing the spirit of Syndrome differentiation, which is one of the most important CTM treatments. Homotherapy for heteropathy means adopting the same treatment in a certain period of different diseases because of the same pathogenesis. Modern medicine indicates that homotherapy for heteropathy might become one of the future medical directions, for there are unreasonable boundaries between one disease and another, whose category is only based on anatomy, but not on the essence of etiology, pathogenesis and pathological changes, etc. Research has shown that different diseases of a similar CTM Syndrome usually have common pathological and physiological changes, so the same theories-diagnostics-prescriptions-drugs can be used as these different diseases might have the same pathological changes at the cellular, molecular or even genetic level, or there exist the same drug target/targets.

Take principles of formulating prescriptions for another example, Huang Shilin, our celebrated CTM expert in the 1980s, designed Compound Huangdai Tablet, a prescription capable of clearing Heat and detoxification as well as invigorating Qi (Vitality) and activating blood, to treat acute promyelocytic leukemia (APL). The prescription was composed of Realgar (Xiong huang), Indigo Naturalis (Qing dai), Salvia Miltiorrhiza (Dan shen) and Radix Pseudostellariae (Tai zi shen). ${ }^{12,13}$ Research has shown that complete remission rate (CRR) of this prescription to APL patients is $96.7 \%$ to $98 \%$, while 5-year disease free survival rate reaches $86.88 \%$, which works efficaciously. Later, it was found in Compound Huangdai Tablet that the main ingredient of Realgar (Xiong huang) was arsenic sulfide (A), and the efficacious ingredient of Indigo Naturalis (Qing dai) and Salvia Miltiorrhiza (Dan shen) was indirubin (I) and tanshinone (T), respectively. Chen Zhu's research team elaborated in detail the mechanism of Compound Huangdai Tablet treating APL from the aspect of molecular biology and biochemistry, which was published in Proceedings of the National Academy of Sciences (PNAS). ${ }^{14}$ Our research on curative efficacy and mechanism of several chronic inflammatory diseases intervened by Kidney (Shen) - invigorating and Qi (Vitality) - strengthening Decoction demonstrated that the prescription made up of Epimedium (Yin yang huo), Radix Astragali (Huang qi) and Radix Rehmanniae (Di huang) could efficaciously intervene chronic inflammation by restoring or improving the inflammatory/antiinflammatory balance (that is, the inflammatory/anti-inflammatory balance adjustment mechanism, IAIBAM), which might provide a direction for further studies concerning modern medicine ${ }^{15-17}$ (Fig. 2).

Meanwhile, the research, recognition and application of bioactive ingredients in Chinese herbal medicines also profoundly show this kind of foreseeability. For example, Artemisia annиa L. (Huang hua hao) was described as far back as 


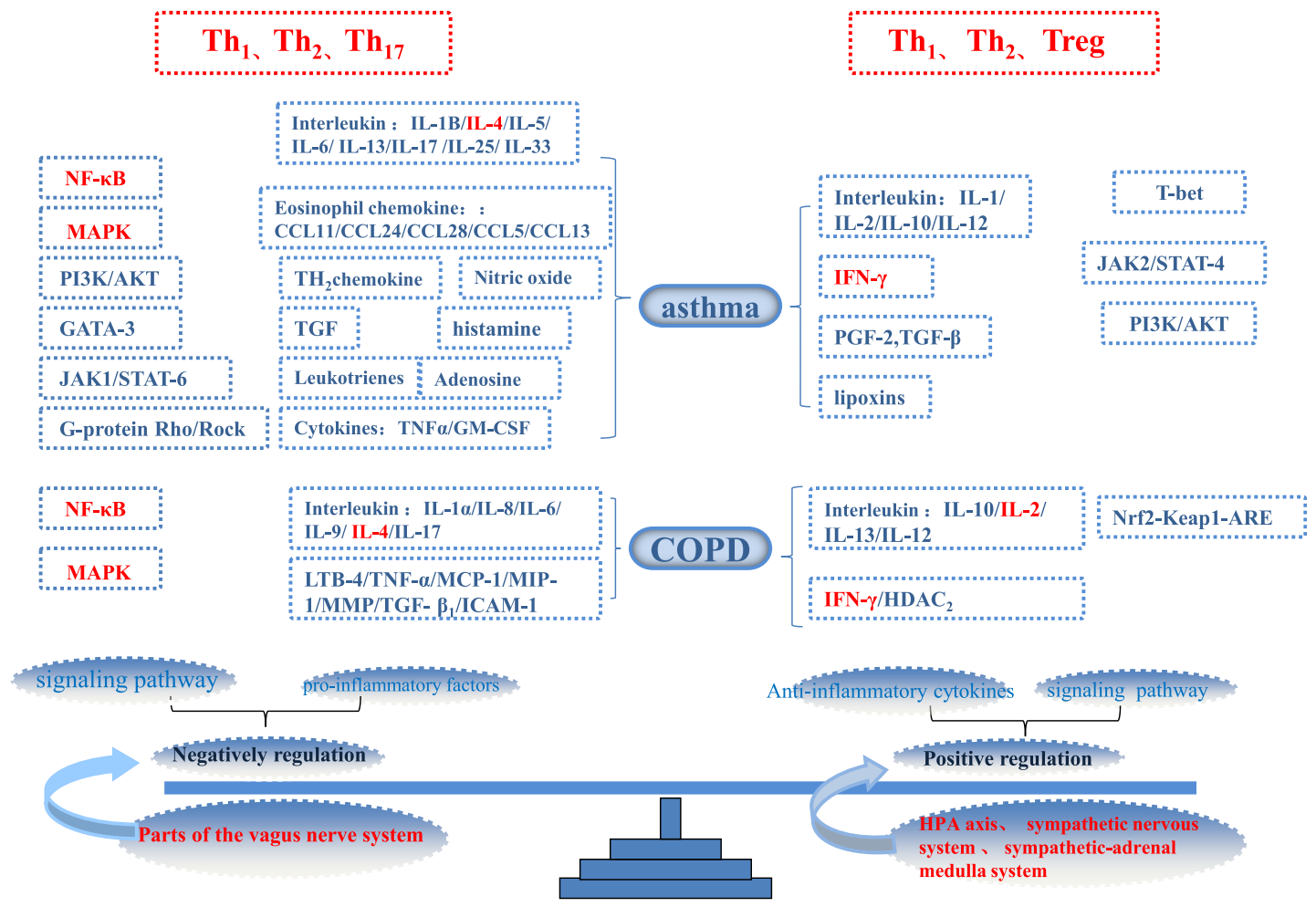

Figure 2. Body adjustment mechanism of inflammatory/anti-inflammatory balance with chronic airway inflammatory diseases.

in Illustrations of Materia Medica (Ben cao tu jing) that it was best at treating fever, which was often used singly in ancient prescriptions. CTM regards that it is capable of clearing Deficieny-Heat, cooling Blood, eliminating hectic fever and preventing malaria. Modern research shows that the main bioactive ingredient of Artemisia аппиа L. (Huang hua hao) is artemisinin that belongs to sesquiterpenoids, which was the first new anti-malaria drug successfully extracted by a Chinese scientist Tu Youyou. ${ }^{18,19}$ This study began under the instruction of Premier Zhou in the mid1960s, with unremittingly in-depth investigations carried out by hundreds of scientists. It was one of the few drugs that was registered internationally and had the autonomous research and development conducted by Chinese scientists, and was estimated by WHO as the only actually efficacious medicine treating pernicious malaria. Before it was discovered, western pharmacologists thought the design of antimalaria drug should begin with nitrogen-containing heterocyclic compounds, while the verification of artemisinin's structure overturned this hypothesis.

Last but not least, take acupuncture for another example. According to different conditions of the body, acupuncture chooses different acupoints and manipulations through the intervention of multiple targets during different periods of a disease, stimulating the body's internal adjustment to reach the aim of treatment. The efficacies of acupuncture manifest not only on the pathological process, but also on the physiological process. It is carried out by the integration of central nervous system, including the reorganization of cortical functions, neural plasticity and the secretion of neurotransmitters and hormones. The mechanism of acupuncture might be the regulation and reorganization directed by central nerves to some specific gene expression so as to treat diseases ultimately. Acupuncture anesthesia is a method by using analgesic effect of acupuncture to relieve pain during operations and prevent physiological disorders, which is similar to anesthesia of modern medicine. It is a good alternative to those who are allergic to anesthetics. Acupuncture analgesia is the interactive function of two different ways to spread stimulation to the brain (spinal cord, brain stem, cerebral cortex, thalamus) in the same spinal ganglion segment from the signal of acupoints and source of pain. Acupuncture stimulates the analgesic substances in the body to resist algogenic substances generated during operations, while analgesic substances such as endogenous opiate-likesubstance participate in acupuncture analgesia and the release of opioid peptides in the brain increases. ${ }^{20}$ Among them, endorphin and enkephalin have strong analgesic effects on the brain, which is positively associated with analgesic effects. ${ }^{21,22}$ There are lots of neurotransmitters participating in acupuncture analgesia, some of which are related to the corresponding central nuclei like the head of caudate nucleus, thalamus centromedian nucleus, raphe nuclei and midbrain central gray matter. Cholecystokinin-8 (CCK-8) is a negative 
feedback mechanism of opioid action, which determines important factors in the effectiveness of acupuncture and morphine analgesia, while individual differences in effectiveness of acupuncture analgesia are determined by relative equilibrium between central opioid peptide and CCK-8. ${ }^{22,23}$ Acupuncture could adjust limbic system reactions caused by stress including hippocampus, cingulate cortex, septum, preoptic area, hypothalamus and amygdale, which might be the physical basis of emotional reactions that acupuncture reduces pain. Different frequencies of electroacupuncture could generate different opioid peptides. For example, a $2-\mathrm{Hz}$ electroacupuncture mainly promoted the release of preproenkephalin (PPE) in the brain, while a $100-\mathrm{Hz}$ electroacupuncture promoted not only the release of preprodynorphin (PPD), but also PPE in some regions of the brain. ${ }^{24}$ Recently, clinical trials of functional magnetic resonance imaging (fMRI) have found that acupuncture is efficacious in reducing chronic pain, which is related to the default network activity of cognitive functions in the brain, further elucidating that acupuncture analgesia works via the central nervous system. ${ }^{25}$

\section{The part that needs to be reconsidered or abandoned}

In the field of medical science, many concepts have changed from absolute to relative ones, and later some have even become fallacy. The application scopes, at the same time, have changed from large to small, which is the inexorable law of development. All the concepts, theorems and laws in natural sciences reflect the stability of things from different aspects. This kind of stability is, to a large extent, conditional and relative, while instability is unconditional and absolute. Any scientific theories have to constantly admit their shortcomings and modify them so as to make progress, giving rise to new concepts, theories and methods. Similarly, modern medicine itself is constantly correcting, improving and developing, continuously searching for the absolute truth. In its hazy period, there used to be lots of extremely simple understandings. For example, before humans were acquainted with the relationship between biology and diseases in 1870s, all the manifestations of inflammation and abscesses were generally described as the attack of toxin, Miasma (Zhang qi), and pathogens. These recognitions are the same as the case in CTM, i.e., simple recognitions due to the limitations of the specific era and culture. In view of this, it is better to adopt the one-dividing-into-two method according to CTM, carrying forward its essence while abandoning its dross.

CTM regards that Heart (Xin) governs Spirit (Shen) while modern medicine has demonstrated that Heart (Xin) in CTM probably refers to brain. Supplements to Invaluable Prescriptions for Diseases (Qian jin yi fang) written by Sun Simiao recorded that mirabilite was salty and bitter in taste, cold in property and non-toxic. Studies of modern medicine have found that there exist nitrogen-containing compounds that would induce hepatocellular carcinoma. Similarly, Asarum forbesii Maxim. (Du heng) was recorded as spicy in taste, a little warm in property and non-toxic. Actually, Asarum forbesii Maxim. (Du heng) contains aristolochic acid that would possibly lead to renal failure, renal cell carcinoma, hepatocellular carcinoma and lymphoma. ${ }^{26-28}$ Prescriptions containing such toxic compounds should be checked and changed because such cases have been discovered in quite a few modern investigations on CTM.

\section{Conclusion}

China is the country that has the most developed traditional medicines around the world, and some of its 56 nationalities have developed their own brilliant traditional medical civilizations, such as Han, Uyghur, Tibetan, Mongolian, Dai, Hui, Miao and Zhuang medicine that have together created the splendid civilizations of CTM and contributed to the prosperity of Chinese people and people all over the world. All of these traditional medicines are the integration of ancient philosophy, clinical experiences, primitive knowledge of medicine, regional cultures and religious beliefs. We should scientifically and rationally recognize CTM, including its concept connotation, philosophical basis, historical status, structure, theories, experience, technology, methods and prescriptions. We should make clear the strengths and weaknesses of CTM through techniques and methods of modern science like methods of epidemiology and evidence-based medicine (EBM), and further demonstrate the scientific basis and effectiveness of its theories, experience, technology, methods and prescriptions. We should further develop the research of effectiveness-demonstrated methods, techniques and prescriptions, make clear the modern scientific connotation of mechanisms as well as the bioactive ingredients in drugs, and launch horizontal comparison of similar theories, diagnostics, prescriptions and drugs. We should uphold the idea of survival of the fittest and preferential development to unveil the real essence of CTM, and promote the development of modern medicine to benefit Chinese people and people all over the world. Only in this way can we make this ancient civilization shine more brilliantly in the contemporary human civilization.

\section{References}

1. Wu B, Chen Y, Huang J, et al. Icariin improves cognitive deficits and activates quiescent neural stem cells in aging rats. J Ethnopharmacol 2012;142:746-53.

2. Qian Y, Li P, Lv B, et al. Heart function and thoracic aorta gene expression profiling studies of ginseng combined with different herbal medicines in eNOS knockout mice. Sci Rep 2017;7:15431. 
3. Ding XF, Chen G, Liu YL, Effects of shengmai for injection on cardiogenic shock. Zhongguo Zhong Yao Za Zhi 2007;32:2298-305.

4. Moey M, Gan XT, Huang CX, et al. Ginseng reverses established cardiomyocyte hypertrophy and postmyocardial infarction-induced hypertrophy and heart failure. Circ Heart Fail 2012;5:504-14.

5. Du CY, Choi RC, Zheng KY, Dong TT, Lau DT, Tsim KW. Yu Ping Feng San, an ancient Chinese herbal decoction containing Astragali Radix, Atractylodis Macrocephalae Rhizoma and Saposhnikoviae Radix, regulates the release of cytokines in murine macrophages. PLoS One 2013;8:e78622.

6. Du CY, Zheng KY, Bi CW, Dong TT, Lin H, Tsim KW. Yu Ping Feng San, an ancient Chinese herbal decoction, induces gene expression of anti-viral proteins and inhibits neuraminidase activity. Phytother Res 2015;29:656-61.

7. Wei P, Huo HL, Ma QH, et al. Pharmacokinetic comparisons of five ephedrine alkaloids following oral administration of four different Mahuang-Guizhi herb-pair aqueous extracts ratios in rats. J Ethnopharmacol 2014;155:642-48.

8. Lin $\mathrm{YC}$, Chang $\mathrm{CW}, \mathrm{Wu} \mathrm{CR}$, Antitussive, anti-pyretic and toxicological evaluation of Ma-Xing-Gan-Shi-Tang in rodents. BMC Complement Altern Med 2016;16:456.

9. Dong J. The relationship between traditional Chinese medicine and modern medicine. Evid Based Complement Alternat Med 2013;2013:153148.

10. Nurahmat M, Chen M, Luo Q, Ling Y, Dong J, Huang C. Rapid characterization and determination of multiple components in Bu-Shen-Yi-Qi-Fang by high-performance liquid chromatography coupled to electrospray ionization and quadrupole time-of-flight mass spectrometry. J Sep Sci 2014;37:3509-17.

11. Li B, Luo QL, Nurahmat M, et al. Establishment and comparison of combining disease and syndrome model of asthma with "kidney yang deficiency" and "abnormal savda". Evid Based Complement Alternat Med 2013;2013:658364.

12. Xiang Y, Wang XB, Sun SJ, et al. Compound huangdai tablet as induction therapy for 193 patients with acute promyelocytic leukemia. Zhonghua Xue Ye Xue Za Zhi 2009;30:440-2.

13. Xiang Y, Chang XH, Cheng YB. Effect of post-remission therapy mainly with compound huangdai tablet on long-term survival of patients with acute promyelocytic leukemia. Zhongguo Zhong Xi Yi Jie He Za Zhi 2010;30:1253-6.

14. Wang L, Zhou GB, Liu P, et al. Dissection of mechanisms of Chinese medicinal formula Realgar-Indigo naturalis as an effective treatment for promyelocytic leukemia. Proc Natl Acad Sci U S A 2008;105:4826-31.
15. Li L, Sun J, Xu C, et al. Icariin ameliorates cigarette smoke induced inflammatory responses via suppression of NF-kappaB and modulation of GR in vivo and in vitro. PLoS One 2014;9: e102345.

16. Wei Y, Liu B, Sun J, et al. Regulation of Th17/Treg function contributes to the attenuation of chronic airway inflammation by icariin in ovalbumin-induced murine asthma model. Immunobiology 2015;220:789-97.

17. Wei Y, Luo QL, Sun J, Chen MX, Liu F, Dong JC. Bu-ShenYi-Qi formulae suppress chronic airway inflammation and regulate Th17/Treg imbalance in the murine ovalbumin asthma model. J Ethnopharmacol 2015;164:368-77.

18. Weathers PJ, Elfawal MA, Towler MJ, Acquaah-Mensah GK, Rich SM. Pharmacokinetics of artemisinin delivered by oral consumption of Artemisia annua dried leaves in healthy vs. Plasmodium chabaudi-infected mice. J Ethnopharmacol 2014;153:732-6.

19. Cai TY, Zhang YR, Ji JB, Xing J. Investigation of the component in Artemisia annua L. leading to enhanced antiplasmodial potency of artemisinin via regulation of its metabolism. J Ethnopharmacol 2017;207:86-91.

20. Lin JG, Chen WL. Acupuncture analgesia: A review of its mechanisms of actions. Am J Chin Med 2008;36:635-45.

21. Bodnar RJ. Endogenous opiates and behavior: 2015. Peptides 2017;88:126-88.

22. Zhao ZQ. Neural mechanism underlying acupuncture analgesia. Prog Neurobiol 2008;85:355-75.

23. Jie W, Fang X, Pu R, et al. Analgesic effect of buccal acupuncture on acute arthritis in rabbits and underlying mechanisms. Zhong Nan Da Xue Xue Bao Yi Xue Ban 2017;42:517-21.

24. Qi D, Wu S, Zhang Y, Li W. Electroacupuncture analgesia with different frequencies is mediated via different opioid pathways in acute visceral hyperalgesia rats. Life Sci 2016;160:64-71.

25. Theysohn N, Choi KE, Gizewski ER, et al. Acupuncturerelated modulation of pain-associated brain networks during electrical pain stimulation: A functional magnetic resonance imaging study. J Altern Complement Med 2014;20:893-900.

26. Xiang T, Yang Z, Sun B, et al. Traditional Chinese medicine: Pivotal role of the spleen in the metabolism of aristolochic acid I in rats is dependent on oatp2a1. Mol Med Rep 2016;14:3243-50.

27. Yu FY, Wu TS, Chen TW, Liu BH. Aristolochic acid I induced oxidative DNA damage associated with glutathione depletion and ERK1/2 activation in human cells. Toxicol In Vitro 2011;25:810-6.

28. Jadot I, Decleves AE, Nortier J, Caron N. An integrated view of aristolochic acid nephropathy: Update of the literature. Int J Mol Sci 2017;18(2):pii: E297. 